\title{
INHOMOGENEOUS QUANTUM GROUPS AS SYMMETRIES OF PHONONS.
}

\author{
F.Bonechi ${ }^{1}$, E.Celeghini ${ }^{1}$, R. Giachetti ${ }^{2}$, E. Sorace ${ }^{1}$ and M.Tarlini $^{1}$. \\ ${ }^{1}$ Dipartimento di Fisica, Università di Firenze and INFN-Firenze, \\ ${ }^{2}$ Dipartimento di Matematica, Università di Bologna and INFN-Firenze.
}

\begin{abstract}
The quantum deformed $(1+1)$ Poincaré algebra is shown to be the kinematical symmetry of the harmonic chain, whose spacing is given by the deformation parameter. Phonons with their symmetries as well as multiphonon processes are derived from the quantum group structure. Inhomogeneous quantum groups are thus proposed as kinematical invariance of discrete systems.
\end{abstract}

PACS 02.20.+b; 02.90.+p; 03.65.Fd

Just from their early setting it has been observed the affinity of quantum groups with finite difference calculus [1] and the presence of $q$-formulas in the realizations of these new algebras has laid the ground for a theory of $q$-special functions [2]. It has also been shown that the quantum geometry associated to the action of $q$-deformed groups on quantum spaces can be given by means of $q$-difference operators [3].

Recently the quantization of inhomogeneous algebras of large physical interest has been introduced $[4,5,6]$ and a relevant point is that the deformation parameter can acquire a dimension. This possibility, realized by using a contraction procedure $[4,5]$, results in a meaningful tool for the description of problems with a fundamental length scale.

In this letter we show that the $q$-deformed pseudoeuclidean algebra $E_{q}(1,1)$ [4] arises quite naturally as kinematical symmetry of phonon propagation in harmonic crystals, whose lattice spacing is interpreted as the dimensional deformation parameter. Even the coalgebra plays a fundamental physical role as it establishes the rules for combining single phonons into composite systems.

E-mail: TARLINI@FI.INFN.IT

DFF 152/12/91 Firenze 
Let us consider the linear chain of equal masses lying at a distance $a$ from one another, with nearest neighbor harmonic interaction and subjected to an external restoring force. The equations of motion are:

$$
\ddot{z}_{j}(t)=\omega^{2}\left(z_{j-1}(t)+z_{j+1}(t)-2 z_{j}(t)\right)-c^{2} z_{j}(t),
$$

where $z_{j}(t)$ is the displacement of the $j$-th point $(j=0,1, \ldots, N)$. Periodic boundary conditions are assumed and initial conditions $z_{j}(0), \dot{z}_{j}(0)$ must be specified.

We embed the ordinary system (1) into the PDE

$$
\left(\partial_{t}^{2}+(2 v / a)^{2} \sin ^{2}\left(-i a \partial_{x} / 2\right)+m^{2}\right) z(x, t)=0,
$$

where $v=\omega a, m=c a$. Letting $L=N a$, the periodic conditions are $z(0, t)=$ $z(L, t)$ while the Cauchy data for (2) consist in the assignment of smooth functions $z(x, 0)$ and $\partial_{t} z(x, 0)$. When $z(j a, 0)=z_{j}(0), \partial_{t} z(j a, 0)=\dot{z}_{j}(0)$ for all $j$, it is easy to see that the solutions of $(1)$ are directly obtained as $z_{j}(t)=z(j a, t)$ irrespectively of the behaviour of the solutions of equation (2) in the points $x \neq j a$.

The continuum limit of (2) obviously reproduces the Klein-Gordon equation in dimension $(1+1)$ with velocity $v$. This constitutes a differential realization of the Casimir of the $E(1,1)$ algebra, which is actually the kinematical symmetry of the continuous system. Likewise, the differential operator in (2) gives a realization of the Casimir invariant of the pseudoeuclidean quantum algebra $E_{q}(1,1)[4]$ with $q=e^{i a}$ and real $a$, which in its own right can be considered the kinematical symmetry of the discrete system.

Indeed, in terms of the generators $P_{0}, J, k, k^{-1}$ and of the unity $\mathbb{I}$ of the algebra, the defining relations of $E_{q}(1,1)$ read

$$
k P_{0} k^{-1}=P_{0}, \begin{aligned}
k J k^{-1} & =J+a P_{0}, \\
J P_{0}-P_{0} J & =\left(k-k^{-1}\right) /(2 a)
\end{aligned}
$$

The coproducts are

$$
\begin{aligned}
& \Delta(k)=k \otimes k \\
& \Delta\left(P_{0}\right)=k^{-1 / 2} \otimes P_{0}+P_{0} \otimes k^{1 / 2} \\
& \Delta(J)=k^{-1 / 2} \otimes J+J \otimes k^{1 / 2},
\end{aligned}
$$

while, for antipodes and counits we have

$$
\begin{gathered}
\gamma(J)=-J-(a / 2) P_{0}, \quad \gamma(k)=k^{-1} \quad \gamma\left(P_{0}\right)=-P_{0}, \\
\epsilon(J)=\epsilon\left(P_{0}\right)=0 \quad \epsilon(k)=1 .
\end{gathered}
$$


From equations (3) and (4) it appears that $k$ is a group-like element of the Hopf algebra $E_{q}(1,1)$. If we write $k=\exp (i a P)$, it is easy to see that $P$ is a primitive element and it is determined up to an integer multiple of $2 \pi / a$. The Casimir operator is well defined in terms of $P$ and is given by:

$$
C=P_{0}^{2}-(2 / a)^{2} \sin ^{2}(a P / 2)
$$

A realization of the relations (3) is obtained from [4], with minor changes, yielding

$$
P_{0}=(i / v) \partial_{t}, \quad k=\exp \left(a \partial_{x}\right), \quad J=i(x / v) \partial_{t}-(v t / a) \sin \left(-i a \partial_{x}\right)
$$

Identifying $C=m^{2} / v^{2}$, the Casimir relation (5) reproduces the Klein-Gordon equation on the lattice $(2)$.

Here we analyze the case with $m=0$, which describes the phonons. In the momentum representation, a realization of the $E_{q}(1,1)$ in terms of the diagonal $P$ and the position operator $X=i \partial / \partial p$ is given by:

$$
\begin{aligned}
& P_{0}=(2 / a) \sin (a p / 2), \quad 0 \leq p<2 \pi / a, \\
& J=(1 / a)\{\sin (a p / 2), X\}_{+}, \\
& P=p
\end{aligned}
$$

The limitation in the values of $p$ permits the reduction to the first Brillouin zone, where $P_{0}$ has positive values.

The expression for the generator $J$ can be inverted in $X$ :

$$
X=(1 / 2)\left\{P_{0}^{-1}, J\right\}_{+}
$$

The time derivative of $X$ is given by $\dot{X}=i v\left[P_{0}, X\right]$ and the commutator, evaluated by the use of (7), gives the well known group velocity of the phonons

$$
\dot{X}=v_{g}=v \cos (a P / 2)
$$

Let us show how the coproduct can be brought to bear to the study of the fusion of phonons. It is well known that, when the symmetry is given by a Lie algebra, the generators of the global symmetry of a composed system are obtained by summing the generators of the symmetry of the elementary constituents. This is related to the fact that each generator $G$ of a Lie algebra is a primitive element, i.e. $\Delta(G)=\mathbb{I} \otimes G+G \otimes \mathbb{I}$. Then $G^{(1)} \equiv G \otimes \mathbb{I}$ acts on the vector space 
of the first elementary system and $G^{(2)} \equiv \mathbb{I} \otimes G$ on the second. The algebras generated by $G^{(1)}$ and $G^{(2)}$ are both isomorphic to that generated by $G$ and since $\Delta$ is a homomorphism of algebras, then $G^{(1)}+G^{(2)}$ generates the same symmetry on the composed system. In the quantum group context we can have non primitive generators, but the very same considerations are still valid. The coproducts (4) induce the global variables $P_{0}=e^{-i a P^{(1)} / 2} P_{0}^{(2)}+P_{0}^{(1)} e^{i a P^{(2)} / 2}$, $J=e^{-i a P^{(1)} / 2} J^{(2)}+J^{(1)} e^{i a P^{(2)} / 2}$ and $k=k^{(1)} k^{(2)}$ so that $P=P^{(1)}+P^{(2)}+2 \pi n / a$, where $n$ can be any integer value and will be chosen to keep $P$ in the fixed Brillouin zone. The composition of the momenta shows that the Umklapp process is implied by the quantum group symmetry.

In concrete, take two differently polarized phonons with the same direction of propagation, velocity parameters $v_{1}$ and $v_{2}$ and dispersion relations (see e.g. [7]):

$$
\Omega_{1}=\left(2 v_{1} / a\right) \sin \left(a P^{(1)} / 2\right), \quad \Omega_{2}=\left(2 v_{2} / a\right) \sin \left(a P^{(2)} / 2\right)
$$

We then have $P_{0}^{(1)}=\Omega_{1} / v_{1}$ and $P_{0}^{(2)}=\Omega_{2} / v_{2}$. The explicit coproduct of $P_{0}$ reads

$$
\begin{aligned}
P_{0} & =e^{-i a P^{(1)} / 2}(2 / a) \sin \left(a P^{(2)} / 2\right)+(2 / a) \sin \left(a P^{(1)} / 2\right) e^{i a P^{(2)} / 2} \\
& =(2 / a) \sin \left(a\left(P^{(1)}+P^{(2)}\right) / 2\right)
\end{aligned}
$$

and the symmetry in the exchange of the two elementary components together with the reality of the result is straightforward. The energy conservation implies the existence of a global velocity $v$ such that, if $\Omega=\left|P_{0}\right| v=(2 v / a) \sin (a P / 2)$, then $\Omega=\Omega_{1}+\Omega_{2}$.

Contrary to energy and momentum, the global boost $J$ is not directly symmetric and, to yield the correct statistics of the composite system, it must be taken in the symmetrized form

$$
J_{s}=\cos \left(a P^{(1)} / 2\right) J^{(2)}+\cos \left(a P^{(2)} / 2\right) J^{(1)}
$$

The generator $J_{s}$ is real and, together with $P_{0}$ and $P$, still closes the $E_{q}(1,1)$ algebra. Moreover from equations (7) to (9) we get the position operator of the composed phonon:

$$
X=\frac{1}{2}\left(X^{(1)}+X^{(2)}\right)+\frac{1}{2}\left\{\frac{\sin \left(a\left(P^{(1)}-P^{(2)}\right) / 2\right)}{\sin \left(a\left(P^{(1)}+P^{(2)}\right) / 2\right)}, \frac{1}{2}\left(X^{(1)}-X^{(2)}\right)\right\}_{+},
$$

which reproduces the Heisenberg algebra $[X, P]=i$ for the global variables. Finally the group velocity of the composite system $\dot{X}=i[\Omega, X]=v \cos (a P / 2)$ 
appears formally identical to that of the elementary system, having performed the Umklapp process.

We conclude by observing that the very same procedure can be applied to the interaction of any number of phonons. Exactly as in the two phonon case it is easily verified that energy and momentum come out in a directly symmetric form. The global boost, first calculated from the coproduct and then completely symmetrized, close again the $E_{q}(1,1)$ algebra.

\section{References.}

[1] V. G. Drinfel'd, Proc. ICM Berkeley, (Providence, R.I., 1986, AMS) 798;

N.Yu. Reshetikhin, L.A. Takhtadzhyan and L.D. Faddeev, Algebra and Analysis 1, 193 (1990).

[2] G.E. Andrews, Reg. Conf. Series in Math. n. 66 (Providence, R.I., 1986, AMS);

L.L. Vaksman and L.I. Korogodskii, Soviet Math. Dokl. 39, 173 (1989);

H.T. Koelink, On quantum groups and q-special function, Ph.D. Thesis, (Leiden, Holland, 1991).

[3] P.P. Kulish and E.V. Damashinsky, J. Phys. A: Math. Gen., 23, L415 (1990);

M. Arik, Z. Phys. C: Particles and Fields, 51, 627 (1991).

[4] E. Celeghini, R. Giachetti, E. Sorace and M. Tarlini, J. Math. Phys. 31, 2548 (1990); J. Math. Phys. 32, 1155 (1991); J. Math. Phys. 32, 1159 (1991);

E. Celeghini, R. Giachetti, E. Sorace and M. Tarlini, "Contractions of quantum groups", Proceedings of the first semester on quantum groups, Eds. L.D. Faddeev and P.P. Kulish, Leningrad October 1990, Springer-Verlag, in press.

[5] J. Lukierski, H. Ruegg, A. Nowicki and V.N. Tolstoy, Phys. Lett B 264, 331 (1991).

[6] V. Dobrev, Canonical q-Deformation of non compact Lie (Super -) Algebras, Göttingen University preprint, July 1991;

J. Lukierski and A. Nowicki, Quantum deformations of D $=4$ Poincaré and Weyl algebra from $q$-deformed $D=4$ conformal algebra, University of Wroclaw preprint, October 1991 ITP UWr 787/91.

[7] N.W.Ashcroft, N.D.Mermin, Solid State Physics, (HRS International Editions, Philadelphia, PA, 1987). 